\title{
NEW INFORMATION RETRIEVAL APPROACH BASED ON SEMANTIC INDEXING BY MEANING
}

\author{
Ala Eddine Kharrat and Lobna Hlaoua \\ Mars Research Lab, Isitcom, University of Sousse, Hammam Sousse, Tunisia
}

\begin{abstract}
An Information Retrieval System (IRS) offers a number of tools and techniques, which enable to locate and visualize the relevant information needed. This information, is expressed by the user in the form of a query natural language. However, the representation of documents and the query in a traditional IRS lead to a lexical-centered relevance estimation which is, in fact, less efficient than a semantic-focused estimation. As a consequence, the documents that are actually relevant are not being recovered if they do not share words with the query, while the documents non relevant, which are words in common with the query, are recovered even though at times they do not have the meaning intended. This paper tackles this problem while suggesting a solution in the level of indexation of an IRS allowing it to improve its performance. To be more precise, we suggest a new approach of semantic indexation allowing to lead to the exact meaning of each term in a document or query undergoing a contextual analysis at the sentence level. In fact, if the system is able to comprehend the need of the user, then consequently it is perfectly capable to respond to it. Add to that, we suggest a simple method allowing to apply any model of IR on our new index table without changing its original bases making it faster. In order to validate this proposed approach, this new created system is evaluated base on numerous collections naming "TIME", "BBC", "The Guardian" and "BigThink". The results based on the experiments indicate the efficacy of our hypothesis compared to traditional IR approaches.
\end{abstract}

\section{KEYWORDS}

Information Retrieval, Indexation, Semantic Index, Exact Word Meaning

\section{INTRODUCTION}

An Information Retrieval System (IRS) is presented as the bridge between the user and a collection of information. Its main objective consists of liking the documents of certain collection with the need of a use in the form of information, which is formed with the help of a query natural language. In order to attend this objective, the IRS is based on certain procedures. As a matter of fact, two main procedures are put in action: The Indexing Process followed by the Research Process. The Indexing Process is the procedure that allows the document or the query to be transferred into a computer representation (a short description or characterization of the content), which reflects its informational content in order to optimize the speed and performance of finding relevant documents to the search. This representation will later be decoded with the help of a model based on a match between the query and the descriptions of a number of documents to measure and evaluate their relevance. Surely enough, numerous models of Information Retrieval (IR) based on distributional calculations are proposed in the literature. These models were widely proven, notably in the treatment in large document collections and very much reliable. The closest model is the vector space model proposed by (Salton et al., 1975) which is based on the distribution of terms in the documents and the query.

In that order, the traditional Information Retrieval System presents a number of gaps at different levels. In fact, the representation of documents (or of the query) most used is the "bag of words" in which the document is considered as a lexical set without considering the issue of ambiguity as a result of the richness of the language. This makes the models unable to consider the fact that an individual term can express multiple significations (polysemy), or that multiple terms can be used to refer to the same term (synonyms). Consequently, the more the document and the query have words in common, the more the document considered relevant. As a result, the relevant documents are not retrieved if they do not share the same words as mentioned in the query, while the non-relevant documents which do share words as mentioned in the query are being 
retrieved even though these words do not share the same significance in the documents and the query. In this context, a number of aspects adapting semantics are proposed in the literature. We can mention the extraction and mapping of concepts, the classification by domains and the recovery of context. It is true that the application of these ideas can help us to better describe the informational content of a document, but it remains a simple estimation which is a bit general and not totally presided.

In order to overcome the lack of elasticity in traditional IR and solve the problem listed previously, we suggest within this paper a new approach of semantic indexation which allows to detect the exact meaning of each term in a plainer and relabel way. This approach is based on the idea of clarifying all the words in relation with each of the term given in order to retreat the most exact sense in a more accurate way. The benefit of this new structure of the index table is that it can be adapted and integrated with any IR model in an easy way without affecting the algorithmic complexity.

This paper is organized as follows. Section 2 presents the related work on Semantic Index and Word Sense Disambiguation. In Section 3, we present our approach. Experimental results are presented in section 4. Finally, Section 5 concludes this paper and shades light on our future work.

\section{RELATED WORK}

Many approaches have been proposed in the literature that were able to well present the content of the document or a query taking into account the ambiguity of words of the natural language. The latter, also called lexical ambiguity, refers to words that are lexically identical even though they have different meanings. It is generally divided into two types (Krovetz et al., 1992) (Krovetz et al., 1997): the syntactic ambiguity and the semantic ambiguity.

- $\quad$ The syntactic ambiguity reflects the words that appear in various grammatical forms. For example, the word "show" in the following phrase "Can you show me the place of the show please?".

- The semantic ambiguity is based on the homonyms and polysemy of the words, which means the words indicate several meanings.

In order to solve the problem of ambiguity in the natural language, and make an information retrieval system capable of perfectly responding to the information needs of users, several workers have operated on the words' meaning disambiguation and on the creation of a semantic index table.

\subsection{Word Sense Disambiguation (WSD) Techniques}

A certain number of techniques of disambiguation of word meanings exist in literature. We can range these approaches into two categories: the approaches based on the external linguistic resources (exogenous approaches), and the approaches based on the learning corpora (endogenous approaches).

Exogenous approaches: these approaches are bases on the use of external linguistic resources in the aim of exploring the general context. We can name the use of Computerized Dictionary or MRD (Machine Readable Dictionnary) (Lesk, 1996) (Veronis et al., 1990) (Nancy et al., 1990) (Wilks et al., 1990) (Guthrie et al., 1991), the use of thesaurus (Yarowsky et al., 1992) and the use of ontologies (Sussna, 1993) (Resnik et al., 1993).

Endogenous approaches: these approaches are based on the usage of a corpus composite of a large number of polysemous words in order to learn useful knowledge about the meaning of the word usage. This identification sentence is called "acknowledge". The issue of this sentence, the disambiguation algorithm is capable to assign the correct term to the words appearing in a sentence based on the knowledge acquired during the learning phase (Weiss, 1973) (Kelly et al., 1975) (Small et al., 1982) (Schütze, 1989).

\subsection{Semantic Indexing Approaches based on Disambiguation}

The objective of the semantic indexing is to represent the documents and the queries with the meaning of their contained words rather than the words themselves. The meanings of words are found with the application of a disambiguation method. As a result, we distinguish:

- The indexing approaches based on endogenous disambiguation (based on the corpus): The principle of these approaches is to first form the necessary knowledge for the disambiguation of words, using a training corpus. The terms of indexing are then treated with the textual content on the 
documents or queries, then disambiguated while being based on the previous acquired knowledge of the sentence. We can name the (Weiss, 1973) approach which is based on the knowledge of the rules of arrangement of operation that are generated with learning from the contexts associated with the different words of a training corpus. We can name the approach of (Schütze et al., 1995) that is based on the degree of recovery of the context of the accuracy of the word intended for disambiguation, and the possible uses of this word in the examined corpus.

- The Indexing approaches based on the exogenous approach (based on the external resources): These approaches are based in general on ontologies in order to determine the different meanings of a word. The basic principle of indexing is then to extract the set descriptor terms of the document. Then, scores are associated with the different meanings found. The meaning with the highest score is then retained as the correct meaning of the corresponding indexing term. We can name the indexing terms of (Baziz et al., 2004) (Khan et al., 2004) (Voorhees, 1993) (Katz et al., 1998) that are based on this idea. Keeping in mind that our approach is also based on this concept.

\section{INTELLIGENT SEMANTIC INDEXING SYSTEM}

\subsection{Motivation}

The main objective of our approach is to create an index table which is based on the semantic comprehension of words. To simplify the idea even more, the aim is to develop a smart indexation system which enables to replace agents. As a matter of fact, to be able to identify the proper meaning of a word, a dictionary, a thesaurus or even an ontology are not enough since the meaning of a word has a close relation with its context. Thereby, we suggest an approach that improves the objective of the classical and even the semantic indexation which focuses on finding a synonym based on ontology towards a system that allows to detect the exact sense of each word in a document or a query while being based on WordNet (Miller, 1995). The idea consists of describing the sense of each phrase consequently the meaning of the document.

\subsection{Description of the Approach}

Our approach is defined throughout 6 main steps: "extended tokenization", "grammatical analysis", "proper meaning detection", "omission of stop words", "stemming \& lemmatization" and "weight assigning". Figure 1 can clarify the general organization of these steps. Following the application of these 6 steps on a document, we obtain a vector of terms: IndexdDocument $=\left\langle t f_{i}: t_{i}, \ldots, t f_{n}: t_{n}\right\rangle$ (with $t f_{i}$ designs the frequency of the term $i, n$ designs the total number of terms). Each term contains its exact meaning. In the case where a term is found more than once in the document but with different meanings, it will be represented in the form of a vector of meanings: $t_{i}=<m f_{i j}: s_{j}, m f_{i(j+1)}: s_{j+1}, \ldots>\left(\right.$ with $m f_{i j}$ designs the frequency of the meaning $\left.s_{j}\right)$.

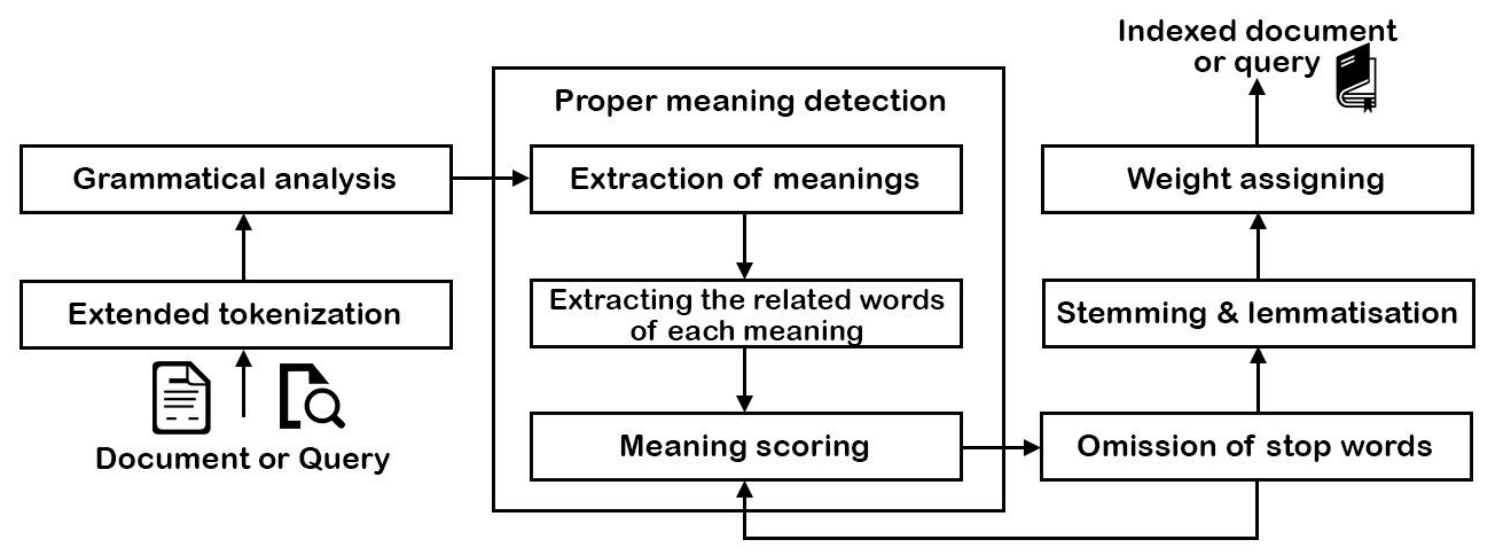

Figure 1. General process of our proposed index 


\subsubsection{Extended Tokenization}

In general, two phrases in a row can have a completely different context. With that in mind, this phase enables to divide the document into a set of sentences in which every sentence obtained will be converted into a certain number of individual words. Next, in order to eliminate the syntactic ambiguity, a very important step permits to detect all collocational words (words that are composed of more than one term like the words "turn off" and "sign in") need to be executed. In our case, every two terms or more in a row, which are available as an entry in WordNet, will be interrupted as collocational words.

\subsubsection{Grammatical Analysis}

This process consists of associating every word of each sentence with their convenient grammatical information like the speech part, the genre, the number, etc. In this context, we use the "TreeTagger" tool developer by (Schmid, 1994). This phase plays an important role in the process of detecting the exact sense of a word. As a matter of fact, the same word can have different meanings completely diverse if we change its position in the phrase. For example, the word "play" as a verb does not have the same sense as the word "play" as a noun. This is also applied with the word "book".

\subsubsection{Proper Meaning Detection}

This step is the main step among all our processes of our approach. It permits, in fact, do detect and describe the exact sense of each word. This step is presented in the form of three sub steps:

Extraction of meanings: this step permits to clarify every possible sense of each word while taking into consideration its grammatical nature. This phase requires the usage of the lexicographical bases of WordNet. The meanings will be ordered according to their usage in the English language, from the most used to the less used. For example, in WordNet, the word "date" has 8 different meanings, starting from the day (being the most common meaning) and finishing with a fruit (being the less common meaning). WordNet, in fact, is an electronic database that covers the majority of nouns, verbs, adjectives and adverbs in the English language. Its objective is to identify, classify and to relate, in various ways, the semantic and lexical content of the English language. This database is constructed in the form of a network of nodes and links. A node, also called "synset", is a number of terms which are synonyms and interchangeable in a context.

Extracting the related words of each meaning: this step consists of extracting all the words that have a relation with each sense of a word. In other words, to find the words that can have a direct or indirect relation with the sense given (the relations of hypernyms, hyponyms, meronym, homonym, toponym, entailment.). The idea is to extract all the words from synets neighbors of the current synset. The words obtained of each meaning will be later on used as an entry for the next step (meaning scoring) in order to detect the sense that shares the most words with the other senses of neighbored words in the phrase.

Meaning scoring: this step allows to order and classify the different senses of each existing word in a sentence in relation to the meanings of the other words in the same sentence. The sense that is classified first will be announced as the most accurate sense. In the case where two senses have the same score, the sense that is most used (according the WordNet) will be classified first (see the algorithm in table 1). The score of each sense will be calculated with the help of the following formula:

$$
\operatorname{Score}\left(s_{i j}\right)=\sum_{k=1 / k \neq i}^{|W|} \sum_{l=1}^{\left|S N_{k}\right|} n b_{-} \operatorname{commun}\left(s_{i j}, s_{k l}\right) * \frac{1}{1+\operatorname{distance}(i, k)}
$$

With $s_{i j}$ presenting the $j^{t h}$ meaning of the word $i, W$ presenting the set of words in a sentence, $S N_{k}$ presenting the set of different meanings of the word $k$, the function $n b_{-}$commun() permits to calculate the number of words in common of the two different meanings and the function distance() calculates the distance between two words in a sentence.

\subsubsection{Omission of Stop Words}

This step consists of filtering and omitting words that do not have any influence on the research process as well as the words that are likely to create poor index terms. Usually, this step is applied in the beginning of the procedure with a classical or semantic representation. However, there is in fact a number of stop words that can be considered as an important element in the research and cannot be omitted from the document or the query (Tong et al., 2008). In this context, in order to avoid this problem, we suggest the following steps: 
- Use the list of words suggested by (Fox, 1989) in order to detect all the stop words in the document or the query. This list consists of 421 words that should be extremely effective in order to filter the most frequent and semantically neutral words in the English literature in general.

- Then, we omit the stop words that do not have any kind of influence on the sentence. It means that these words have a very low score (default score) and does not share words with the other neighbored words in the sentence. If a word has a very important score and shares words with the neighbored words, it will not be omitted.

- In the case where it excites at least a word omitted in a sentence, the previous step (Meanning scoring) will be applied one more time on the same phrase. We move on to the next step if no other stop word is eliminated.

Table 1. Sense Scoring Algorithm

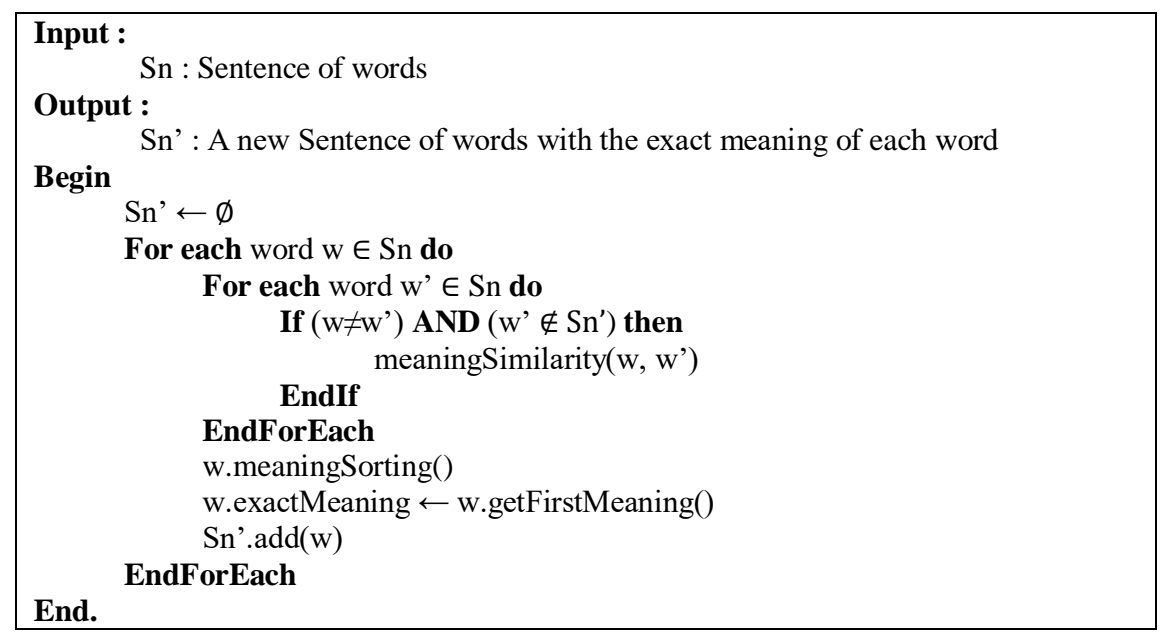

\subsubsection{Stemming \& Lemmatization}

This step enables to substitute a word in order to find its origin. With prudence, it should be applied following the detection of exact sense of each word in a document or a query. In fact, the lemmatization can cause us to lose the sense of a word. For example, the word "player" in English has a different meaning in relation with the meaning of its origin (the word "play"). In literature, the most used lemmatization algorithm for information search applications in a large number of languages is the one of Porter (Porter, 1980). Nevertheless, this algorithm possesses certain gaps for some words, that is because these simple rules can't completely describe English morphology (for example the word "family" is converted to "family"). There is also another major problem, most of the lemmatization algorithms cannot treat the new words or brand names.

In order to avoid such problems, we use an improved version of Porter stemming algorithm which is proposed by (Karaa, Gribâa, 2013) with the algorithm "LemmaGen" (Juršic et al., 2010). Then, the obtained results for each set of word will be tested by WordNet; if it has an entry in WordNet, it will be noted as a well stemmed word. If not, the original word will be treated with the algorithm of WordNet Stemmer. In the case where WordNet Stemmer does not approve the word, this word will not go under any modification. In fact, we want to start from the idea that stemming shouldn't be adopted blindly.

\subsubsection{Weight Assigning}

In this phase, each word will be assigned with its proper degree of importance in the documents. The idea consists of calculating the number of occurrences of each word in all the document, taking into consideration the exact meaning of each word. In the case where a word is being repetitive more than once in a document with different senses, all these senses will be added in an index table and ordered accordingly to their number of appearances. As a total, this step permits the construction of a vector of words for each document. Each word will have its frequency as well as its different meanings presented in the document ordered according to their number of appearances in the document in a general way. 


\subsection{Illustration of the Approach}

Supposedly we have a document containing 5 sentences completely different when it comes to meaning and context: Document $=\{\mathrm{I}$ bow down to pray on the bow of the ship. Winter is my best season. You might season a chicken before roasting. Can you hold the racket please? The party next-door is making a racket.\}

According to this example, our system will firstly separate each sentence apart (extended tokenization) in order to detect the grammatical information of each word in each sentence (grammatical analysis). For example, in the first sentence, the first word "bow" is a verb and the second one is a noun. This analysis actually helps to extract the different meanings of each word in a very precise way. In fact, the word "bow" as a noun has 9 different meanings, and 5 different meaning as a verb. After that, the system will give a score to each meaning of each word to detect its exact meaning (proper meaning detection). Referring again to the first sentence of the example, our system will be able to detect that the first word "bow" means "bend one's knee or body", and the second one means "front part of a vessel or aircraft". As a matter a fact, our system is able to recognize the first word "bow" by relating it to the word "down" and the word "pray". When it comes to the second word "bow" the system was able to recognize it by establishing a relationship with the word "ship". Once the system applies the step of omission of words, we obtain these words: "bow, down, pray, bow, ship". Supposedly the word "down" would be emitted at this stage but our system kept it since it was recognized as an important word to identify the necessary meaning of another word. Finally, we will be converting these words to their original form if needed (Stemming \& lemmatization) counting their frequency in the document.

\section{EXPERIMENTAL EVALUATION}

\subsection{Test Environment}

In order to insure a very rich collection of documents, we decided to collect documents from different resources. In this regard, we collected documents that belong to well-known daily newspapers: "TIME": (http://time.com/), "BBC": (https://www.bbc.co.uk/), "TheGauardian": https://www.theguardian.com and "BigThink": (https://bigthink.com/). Thereby, our dataset contains exactly 3562 documents tackling different themes and domains. In fact, these journals are known by the heterogeneity subjects of their content. This means that the articles of these journals take part of different categories. We can name: "Entertainment", "Technology", "Business", "News", "Culture", "Arts", "Food", "Travel", "Lifestyle”, "Science” etc.

Then we chose a list of queries (20 queries to be exact) that can form a considerable number of relevant documents. Indeed, each query chosen has at least a minimum of 20 relevant documents and a minimum of 50 non-relevant documents that share terms with the query. The idea is to test if our approach is capable of assisting any kind of information research system to detect and omit the noise (the non-relevant documents) while being well based on the exact sense of each word in the document or the query.

With the intention of evaluating and testing efficiency of our indexation system in a correct and reliable manner, it is important to make our new indexation system easily applied on any kind of IR model. Therefore, it is just enough to go through a fundamental step that allows to filter all relevant documents according to the meaning in relation to a given query. This means, omitting all the documents possessing words in common with the query, but do not share the same meaning of words of the query. Then, execute the IR model on the remaining documents (the relevant documents). This idea makes it possible to increase the precision on an IRS (decrease the noise) and eventually permits to decrease the cost of treating in IR model. As a matter of fact, the IR model is only going to treat $n^{\prime}$ documents (the documents obtained following the filtering) instead of treating $n$ documents (the documents indicated as relevant before the filtering) (with $n^{\prime} \leq n$ ).

\subsection{Experiments}

The objective of our experiment is mainly to determine if the application of the new index table with random models of IR equally perfect its performances. In other words, ameliorate the precision without losing the recall. In this matter, we have applied, each time, an IR model on the collection previously presented by relying on the use of the classical indexation table then the index table that is generated by our system. The idea is to 
compare the obtained results of each IR model by using the classical indexation table relative to using the new index table. The IR models used are: the vector space model (Salton et al., 1975) and the probabilistic model (Robertson, 1977). The evaluation of the comparison is affected according to the MAP Measure (Meaning average precision): P@5,P@10,P@15 and P@20. The obtained evaluation results for these different models are presented in the figure 2. According to these results, we noticed the use of the index table of our proposed system with both models give much better results than the models' results that use traditional indexation. Table 2 define the improvement rate of each model using our new indexing system. In fact, we note that a better precision is insured in the case where we apply our indexation approach with a vector space model, but this does not eliminate the fact that the probabilistic model has witnessed, in recent times, a noticeable improvement. In both cases, we note that our approach enables to maintain certain stability in the level of precision (p@5, p@10,p@15 and p@20) for a given model, in contrary of the case of the traditional indexation whether it comes by term or by semantics.

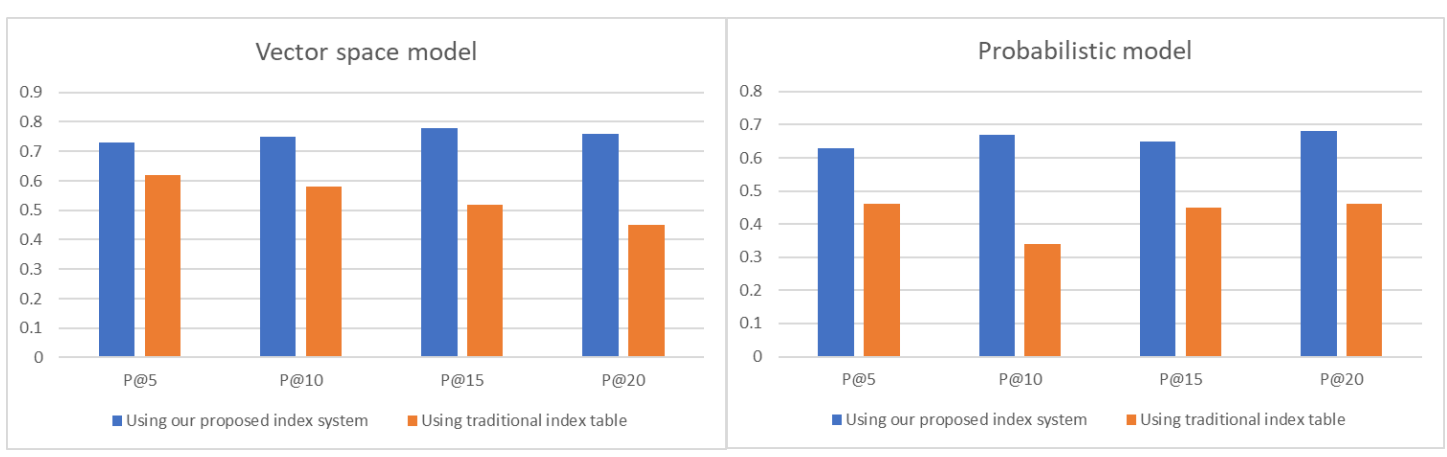

Figure 2. Using our proposed indexing system VS using traditional index

Table 2. The improvement rate of each model using our new indexing system

\begin{tabular}{lcccccccc}
\hline & \multicolumn{3}{c}{ Vector space model } & \multicolumn{4}{c}{ Probabilistic model } \\
\hline MAP Measure & $\mathbf{P} @ \mathbf{5}$ & $\mathbf{P} @ \mathbf{1 0}$ & $\mathbf{P} @ \mathbf{1 5}$ & $\mathbf{P} @ \mathbf{2 0}$ & $\mathbf{P} @ \mathbf{5}$ & $\mathbf{P} @ \mathbf{1 0}$ & $\mathbf{P} @ \mathbf{1 5}$ & $\mathbf{P} @ \mathbf{2 0}$ \\
Traditional Index & 0.62 & 0.58 & 0.52 & 0.45 & 0.46 & 0.34 & 0.45 & 0.46 \\
Proposed Index & $\mathbf{0 . 7 3}$ & $\mathbf{0 . 7 5}$ & $\mathbf{0 . 7 8}$ & $\mathbf{0 . 7 6}$ & 0.63 & 0.67 & 0.65 & 0.68 \\
Improvement rate & $17.74 \%$ & $29.31 \%$ & $50 \%$ & $68.89 \%$ & $\mathbf{3 6 . 9 6 \%}$ & $\mathbf{9 7 . 0 6 \%}$ & $\mathbf{4 4 . 4 4 \%}$ & $\mathbf{4 7 . 8 3 \%}$ \\
\hline
\end{tabular}

These results showed very well that our approach functions perfectly. It is therefore obvious to state that our indexing system is capable of generating a smart index table enabling us to detect the exact meaning of each word in a document or even in a query. Consequently, we can confirm the following hypothesis: "The understanding of a user's need for information has a direct and determinant impact on the performance of an SRI”.

\section{CONCLUSION}

In this paper, we have proposed, for an information retrieval system, a new approach of creating an index table that relies on the semantic comprehension of words. This index table is capable of detecting the exact meaning of each word in a document or even a query. In this order, we can describe the exact meaning of each phrase and therefore the meaning of the document. The structure of this index table allows to make the table itself adaptable and integrable with any IR model with an easy manner without touching the algorithmic complexity. Our experimental results have well shown that this new indexation approach functions perfectly and provides good quality of results for a greatly rich collection of documents that tackles various themes and domains.

It is true that our system has successfully proven its efficiency with multiple IR models, we might as well, in our future work, create our own semantic model that will be more efficient with our proposed index. In other words, a model that has a more profound understanding of the structure of our index table, which will allow it to showcase the user's need for information in a better way. 


\section{REFERENCES}

Baziz, M., Boughanem, M. and Aussenac-Gilles, N., 2004. The use of ontology for semantic representation of documents. In The 2nd Semantic Web and Information Retrieval Workshop (SWIR), SIGIR (pp. 38-45).

Fox, C., 1989, September. A stop list for general text. In Acm sigir forum (Vol. 24, No. 1-2, pp. 19-21). ACM.

Guthrie, J.A., Guthrie, L., Wilks, Y. and Aidinejad, H., 1991, June. Subject-dependent co-occurrence and word sense disambiguation. In Proceedings of the 29th annual meeting on Association for Computational Linguistics (pp. 146-152). Association for Computational Linguistics.

Juršic, M., Mozetic, I., Erjavec, T. and Lavrac, N., 2010. Lemmagen: Multilingual lemmatisation with induced ripple-down rules. Journal of Universal Computer Science, 16(9), pp.1190-1214.

Karaa, W.B.A. and Gribâa, N., 2013. Information retrieval with porter stemmer: a new version for English. In Advances in computational science, engineering and information technology (pp. 243-254). Springer, Heidelberg.

Katz, B., Uzuner, O. and Yuret, D., 1998. Word sense disambiguation for information retrieval. MIT Artificial Intelligence Laboratory Research Abstracts, September 2000.

Kelly, E.F. and Stone, P.J., 1975. Computer recognition of English word senses (Vol. 13). North-Holland.

Khan, L., McLeod, D. and Hovy, E., 2004. Retrieval effectiveness of an ontology-based model for information selection. The VLDB Journal - The International Journal on Very Large Data Bases, 13(1), pp.71-85.

Krovetz, R. and Croft, W.B., 1992. Lexical ambiguity and information retrieval. ACM Transactions on Information Systems (TOIS), 10(2), pp.115-141.

Krovetz, R., 1997, July. Homonymy and polysemy in information retrieval. In Proceedings of the eighth conference on European chapter of the Association for Computational Linguistics (pp. 72-79). Association for Computational Linguistics.

Lesk, M., 1996. Automated sense disambiguation using machine-readable dictionaries: How to tell a pine cone from an ice cone. In Proc. of the SIGDOC Conference.

Miller, G.A., 1995. WordNet: a lexical database for English. Communications of the ACM, 38(11), pp.39-41.

Nancy, I. and Véronis, J., 1990. Mapping dictionaries: A spreading activation approach. In 6th Annual Conference of the Centre for the New Oxford English Dictionary (pp. 52-64).

Porter, M.F., 1980. An algorithm for suffix stripping. Program, 14(3), pp.130-137.

Resnik, P.S., 1993. Selection and information: a class-based approach to lexical relationships. IRCS Technical Reports Series, p.200.

Robertson, S.E., 1977. The probability ranking principle in IR. Journal of documentation, 33(4), pp.294-304.

Salton, G., Wong, A. and Yang, C.S., 1975. A vector space model for automatic indexing. Communications of the ACM, 18(11), pp.613-620.

Schmid, H., 1994. Probabilistic Part-of-Speech Tagging Using Decision Trees, Intl. In Conference on New Methods in Language Processing. Manchester, UK.

Schütze, H. and Pedersen, J.O., 1995. Information retrieval based on word senses.

Schütze, H., 1998. Automatic word sense discrimination. Computational linguistics, 24(1), pp.97-123.

Small, S. and Rieger, C., 1982. Parsing and comprehending with word experts (a theory and its realization). Strategies for natural language processing, pp.89-147.

Sussna, M., 1993, December. Word sense disambiguation for free-text indexing using a massive semantic network. In Proceedings of the second international conference on Information and knowledge management (pp. 67-74). ACM.

Tong, S., Lerner, U., Singhal, A., Haahr, P. and Baker, S., Google LLC, 2008. Locating meaningful stopwords or stop-phrases in keyword-based retrieval systems. U.S. Patent 7,409,383.

Veronis, J. and Ide, N.M., 1990, August. Word sense disambiguation with very large neural networks extracted from machine readable dictionaries. In Proceedings of the 13th conference on Computational linguistics -Volume 2 (pp. 389-394). Association for Computational Linguistics.

Voorhees, E.M., 1993, July. Using WordNet to disambiguate word senses for text retrieval. In Proceedings of the 16th annual international ACM SIGIR conference on Research and development in information retrieval (pp. 171-180). ACM.

Weiss, S.F., 1973. Learning to disambiguate. Information Storage and Retrieval, 9(1), pp.33-41.

Wilks, Y., Fass, D., Guo, C.M., McDonald, J.E., Plate, T. and Slator, B.M., 2007. Providing Machine Tractable Dictionary Tools. In Words and Intelligence I (pp. 167-216). Springer, Dordrecht.

Yarowsky, D., 1992, August. Word-sense disambiguation using statistical models of Roget's categories trained on large corpora. In Proceedings of the 14th conference on Computational linguistics -Volume 2 (pp. 454-460). Association for Computational Linguistics. 